\title{
Dysregulation of dopamine and pathology of prefrontal neurons: neuroimaging studies in schizophrenia and related animal models
}

\author{
ALESSANDRO BERTOLINO
}

\section{INTRODUCTION}

Prior evidence involving dopamine and the prefrontal cortex in the pathophysiology of schizophrenia

Several brain abnormalities have been claimed in schizophrenia, but the pathophysiology of the disorder remains unclear. Of the many reported findings, two lines of research data have emerged as particularly consistent, involving pathology of dorsolateral prefrontal cortex and dysregulation of the dopamine system. Abnormal dorsolateral prefrontal cortical function has been implicated in a number of neuropsychological and neuroimaging studies (Weinberger et al. 1986; Weinberger, 1987; Braff et al. 1991; Goldberg \& Gold, 1995; Andreasen et al., 1994; Frith et al., 1995, Callicott et al., 1998). Moreover, post mortem tissue studies have found evidence of subtle pathology of dorsolateral prefrontal glutamatergic neurons and of their connectivity (Selemon et al. 1995; Lewis, 1997; Perrone-Bizzozzero et al., 1996; Rajkowska et al., 1998). On the other hand, while involvement of dopamine in the pathophysiology of schizophrenia is uncontested, the true neurobiological mechanisms underlying its dysregulation are still unclear. The «dopaminergic hypothesis» of schizophrenia, that suggested hyperactivity of central dopamine neurons, was based on indirect pharmacological evidence (Carlsson, 1988); for example, dopamimetic drugs such as amphetamine have psychotogenic properties and antipsychotic drugs, such as chlorpromazine, are dopamine antagonists. Efforts to measure directly dopamine concentrations,

Indirizzo per la corrispondenza: Professor A. Bertolino, Clinical Brain Disorders Branch, National Institute of Mental Health, Bethesda MD (USA).

Fax: +1-301-402.0743.

E-mail: bertolia@intra.nimh.nih.gov metabolic enzymes, and receptor protein abundance in post mortem tissue as well as dopamine receptor binding with in vivo neuroimaging techniques have been largely inconclusive, dimming enthusiasm for the traditional dopamine hypothesis (Hietala et al., 1994; Kornhuber et al. 1989; Lee et al., 1978; Knable et al., 1997). However, three recent studies using $\mathrm{D}_{2}$ radioreceptor imaging have found that amphetamine causes larger displacement of the ligand from striatal receptors in schizophrenia than in healthy subjects, suggesting greater release of dopamine following amphetamine challenges (Laruelle $e t$ al., 1996; Abi-Dargham et al., 1998; Breier et al., 1997). These data have reinvigorated interest in a dopamine abnormality, not in terms of «too much dopamine" per se, but in more subtle terms of abnormal responses. of dopamine neurons to stimuli.

Is pathology of neurons in prefrontal cortex associated with dysregulation of dopamine?

Until relatively recently, the possibility that pathology of dorsolateral prefrontal cortex and dopamine dysregulation might be directly linked neurobiological phenomena has received little attention. In this regard, evidence from experiments in rodents, has indicated that the prefrontal cortex through glutamatergic projections (Divac et al., 1978) regulates the burst firing patterns (Svensson \& Tung, 1989; Murase et al., 1993) and the number of spontaneously active dopamine neurons in the ventral brainstem (the neurons projecting to prefrontal cortex and to the striatum) (Shim et al., 1996). Moreover, it has been demonstrated that prefrontal lesions exaggerate the release of subcortical dopamine evoked by drug challenges (including amphetamine) as well as by stress (Braun et al., 1993; Jaskiw et al., 1990; Flores et al., 1996; Roberts et al., 1994). 
Such observations have led to speculation that prefrontal pathology in schizophrenia may lead to secondary dysregulation of subcortical dopamine activity, thus explaining dopamine related symptoms as resulting primarily from disordered cortical function (Weinberger, 1987; Jaskiw \& Weinberger, 1992; Davis et al., 1991; Deutch, 1992).

An elaboration of this hypothesis was proposed by Grace (1991), who anticipated from studies in rodents that a decrease in dorsolateral prefrontal cortex function in schizophrenia might lead to a reduction in tonic dopamine release. A corollary of this hypothesis is that in the context of such reduced tonic or steady-state dopamine activity, the response of dopamine neurons to phasic events, e.g. the depolarization of dopamine neurons in response to behaviorally relevant or to pharmacological stimuli (Grace, 1991; Schultz et al., 1997), might be exaggerated, perhaps accounting for the exacerbation of symptoms and clinical decompensation that often occur in patients during stress.

\section{NEUROIMAGING EXPERIMENTS}

\section{Brief introduction to the techniques used}

In our laboratory, we have performed several studies with the purpose of investigating the relationship between neuronal integrity of dorsolateral prefrontal cortex and steady-state and stimulus-induced striatal dopamine activity in patients with schizophrenia. We have assessed neuronal integrity in vivo using proton magnetic resonance spectroscopic imaging ( ${ }^{1} \mathrm{H}$-MRSI) which provides measures of $\mathrm{N}$-acetylaspartate (NAA). We have instead assessed dopamine activity in vivo using $\mathrm{D}_{2}$ radiotracer imaging techniques that allow measurement of steady-state and stimulus-induced release of subcortical dopamine.

Without going into many details, in the mature brain (when glial and neuronal cells are completely differentiated), NAA is found exclusively in neurons and in highest concentrations in pyramidal glutamatergic neurons (Urenjak et al., 1993; Moffet \& Namboodiri, 1995). NAA synthesis takes place in the mitochondria, it is ADP dependent and uses glutamate as a precursor (Bates et al., 1996). While the biological role of NAA has yet to be clearly defined, it acts via the glutamatergic NMDA receptor to elevate intracellular calcium (Rubin et al., 1995); its concentrations are reduced by pharmacological inhibition of mitochondrial energy metabolism; its reductions correlate highly with the relative reduction of ATP and $\mathrm{O}_{2}$ consumption (Bates et al., 1996). Moreover, a number of studies have demonstrated that NAA reductions are reversible suggesting that NAA is sensitive to pathological processes affecting the function of neurons (Clark, 1998). Based on the above evidence, it has also been speculated that, at least in the context of pathology, NAA measures may vary as a correlate of glutamatergic activity of neurons. A number of studies in schizophrenia have shown that NAA relative concentrations are reduced in the dorsolateral prefrontal cortex and in mesial temporal limbic structures (including the hippocampus) suggesting regionally specific neuronal pathology in these two regions (Buckley et al., 1994; Nasrallah et al., 1994; Renshaw et al., 1995; Maier et al., 1995; Bertolino et al., 1996; 1998a, b, c; Deicken et al., 1997).

Radiotracer imaging techniques such as Positron Emission Tomography (PET) and Single Photon Emission Computed Tomography (SPECT) use similar technical principles for the study of dopamine receptors. The radiotracers we have used are ${ }^{123} \mathrm{IBZM}$ for the SPECT study and ${ }^{11} \mathrm{C}$-Raclopride for the PET experiment (see below). These tracers have high selectivity and low affinity for dopamine $D_{2} /$ $\mathrm{D}_{3}$ receptors and compete with synaptic dopamine for receptor occupancy (Breier et al., 1997). These techniques can be used for measuring steady-state and simulus-induced activity of dopamine. For the steady-state measurement, once infused, the tracer will bind to free receptors, whereas those receptors occupied by dopamine will remain untouched. Therefore, the measured radioactivity will represent the density or affinity of the free receptors and, by inference, it will allow indirect measurement of the steady-state activity of dopamine. For the stimulusinduced experiments two infusions of tracers are performed. With the first, one can measure the steadystate activity. After the first infusion of radiotracer, the stimulus is provided (the stimulus can be pharmacological or of other nature) and then a second infusion of radiotracer is performed. Since these tracers have low affinity for the receptors, they will be easily displaced by the stimulus-released dopamine. Therefore, by subtracting the radioactivity measured after the stimulus from the radioactivity measured at steady-state obtained in the same experiment, it is possible to indirectly measure stimulus-induced release of dopamine. 


\section{A. Bertolino}

Evidence for the association between prefrontal pathology and dopamine activity in schizophrenia

Based on evidence that dopamine neurons are modulated by excitatory inputs from prefrontal cortical neurons and in agreement with Grace's theory, we hypothesized that compromised neuronal integrity in dorsolateral prefrontal cortex would predict low basal dopamine activity. In further agreement with Grace, we also hypothesized that dysfunctional inputs from prefrontal neurons would predispose dopaminergic neurons to exaggerated stimulus-induced responses.

In the first experiment (Bertolino et al., 1999), we assessed the relationship between striatal dopamine receptor radiotracer binding (as assessed with ${ }^{123}$ IBZM-SPECT) and dorsolateral prefrontal cortex neuronal integrity (as assessed with ${ }^{1} \mathrm{H}$-MRSI measures of NAA). We studied 14 patients with schizophrenia who had been drug-free for at least two weeks. The results of this study showed a negative correlation between NAA relative concentration in dorsolateral prefrontal cortex and steady-state striatal ${ }^{123}$ IBZM binding in the patients. No correlation was found for NAA relative measures in any of the other cortical regions sampled. Therefore, these data suggest that dorsolateral prefrontal cortex neuronal integrity is selectively predictive of steady-state levels of striatal dopamine activity in patients with schizophrenia. The negative correlation between NAA measures and $\left[{ }^{123} \mathrm{I}\right]$ IBZM binding to dopamine receptors suggests that the lower is the NAA, the higher is the binding. In other words, as hypothesized, more neuronal pathology in the dorsolateral prefrontal cortex is correlated to higher binding of the tracer and, by inference, to lower steady-state activity of dopamine.

In the second experiment (Bertolino et al., submitted for publication), we assessed the relationship between striatal stimulus-induced release of dopamine (as assessed with ${ }^{11} \mathrm{C}$-Raclopride PET) and neuronal integrity in dorsolateral prefrontal cortex of eight patients with schizophrenia (four drug-naive and four drug-free for at least two weeks) and eight healthy controls. To study stimulus-induced release of dopamine in these subjects, we infused amphetamine (which causes a large release of monoamines and in particular of dopamine) between the two infusions of radiotracer (see above). We found a selective negative correlation between NAA measures in dorsolateral prefrontal cortex and changes in striatal raclopride binding after amphetamine in- fusion in patients with schizophrenia. Lower NAA measures in dorsolateral prefrontal cortex predicted greater decrease in raclopride binding, likely caused by increased amphetamine-induced release of dopamine. No other region in the patients or NAA measures in dorsolateral prefrontal cortex of healthy subjects showed this correlation, underlining the regional specificity of this relationship and its association with schizophrenia. Therefore, as hypothesized, these data demonstrate that the apparent increased release of striatal dopamine after amphetamine infusions in patients with schizophrenia is related to NAA measures in dorsolateral prefrontal cortex.

Evidence for the association between prefrontal pathology and dopamine activity in animal models of schizophrenia

As a test of the interpretation of our results in patients with schizophrenia, we performed further experiments in an animal model of schizophrenia. Maldevelopment of dorsolateral prefrontal cortex is a popular hypothesis concerning the pathophysiology of schizophrenia (Lewis, 1997; Weinberger \& Lipska, 1995). Simplistically, these authors have hypothesized that altered development of the connectivity of prefrontal cortex, possibly due to primary lesions in the hippocampal area, can be responsible of the pathophysiology of schizophrenia. In this regard, we have previously shown that rhesus monkeys with developmental temporal limbic damage (including the hippocampus) grow up to have abnormalities of dorsolateral prefrontal cortex (Bertolino et al., 1997; Saunders et al., 1998) analogous to earlier findings in rats with developmental limbic damage (Weinberger \& Lipska, 1995). Therefore, we explored further the interpretation of our findings in patients with schizophrenia in a non human primate model of developmental prefrontal pathology in which we examined directly the in vivo relationship between NAA measures in dorsolateral prefrontal cortex and dopamine release in striatum. In this case the activity of dopamine was measured directly with in vivo microdialysis. Dopamine activity was measured directly in the striatum at steady-state and after infusion of amphetamine directly in the prefrontal cortex of these monkeys (to simulate the physiological stress-induced release of dopamine). Consistent with our interpretation of the human findings, we found a positive correlation between NAA measures in dorsolateral prefrontal cortex 
and steady-state striatal dopamine activity. In other words, consistent with our findings in schizophrenia, at steady-state monkeys with lower NAA in dorsolateral prefrontal cortex have lower striatal dopamine release. Thus, our data in rhesus monkeys directly support our interpretation of the $\left[{ }^{123} \mathrm{I}\right] \mathrm{IBZM}$ data in our patients with schizophrenia and are consistent with evidence that brainstem dopamine neurons are under tonic control of prefrontal glutamatergic projection neurons (Karreman \& Moghaddam, 1996).

We also tested the other part of the hypothesis in our monkeys by examining the relationship between NAA measures in prefrontal cortex and striatal dopamine release after acute infusion of amphetamine directly into the sulcus principalis of dorsolateral prefrontal cortex (Bertolino et al. 1999). Strikingly, the direction of the correlation was inverted as compared to the steady-state relationship. Thus, after a pharmacological challenge of dorsolateral prefrontal neurons, lower NAA is related to higher striatal dopamine release, also consistent with the hypothesis that abnormal dorsolateral prefrontal cortex integrity leads to dissociated steady-state and stimulusinduced dopamine responses. Our data in monkeys further suggest that maldevelopment of the dorsolateral prefrontal cortex, at least as induced indirectly in our paradigm by a neonatal mesial temporal-limbic lesion and as demonstrated by our previous ${ }^{1} \mathrm{H}$ MRSI (Bertolino et al., 1997) and dialysis studies of these animals (Saunders et al., 1998), is an important factor in the altered responses of dopamine neurons to cortical stimuli. This latter point also may have implications for schizophrenia, as abnormal development of connections between dorsolateral prefrontal cortex and temporal-limbic structures has been implicated (Lewis, 1997; Weinberger \& Lipska, 1995) and stress often leads to an exacerbation of the illness. Very recently, we have further explored in rats the relationship between neuronal development of the prefrontal cortex and dopamine-related activity (Bertolino et al., unpublished data). In these experiments we have found that both NAA in the prefrontal cortex and dopamine related activity are not altered before puberty in rats with developmental lesions of the hippocampus. On the contrary, after puberty (similarly to the post-pubertal onset of schizophrenia) these rats show remarkable deficits of NAA in the prefrontal cortex and temporally associated altered dopamine activity.

Despite the similarities between the data in animals and in patients with schizophrenia there are some caveats. It is clear that patients with schizophrenia do not have a temporal-limbic lesion analogous to what we have produced in our monkeys or rats. This is the principal limitation of interpreting the animal findings in the context of schizophrenia. However, our model of temporal-limbic damage is not aimed at reproducing the lesion that may cause schizophrenia, which has yet to be defined. We have emphasized that our model appears to interfere with the maturation of dorsolateral prefrontal cortex (as already shown in these same monkeys, Bertolino et $a l ., 1997$ ), presumably by damaging connections (direct and indirect) with temporal-limbic structures. It is the effect of our developmental lesion on the function of dorsolateral prefrontal cortex that appears to have face and predictive validity in modeling some aspects of schizophrenia.

It is also important to emphasize that our correlational findings do not prove a causal relationship between the investigated phenomena (i.e. dorsolateral prefrontal cortex and striatal dopamine). However, there are two unique and striking aspects of the correlations which argue for their significance. First, they involve two brain systems widely believed to be crucial to understanding schizophrenia, i.e. dorsolateral prefrontal cortex and dopamine, and they are related in a neurobiologically plausible way, consistent with hypotheses from earlier preclinical studies. The second striking aspect of the correlations is that they are regionally specific, i.e. found only between brainstem dopamine activity and neurons of the dorsolateral prefrontal cortex. The fact that both correlations are exactly as predicted from basic anatomical and electrophysiological studies is also noteworthy. Notwithstanding the limitations of a correlational study, it is an experiment that addresses the relationships of varying phenomena in different systems in the living brain, such as a population of neurons in the dorsolateral prefrontal cortex and dopamine activity in the striatum.

\section{CONCLUSIONS}

The results presented in this review paper provide the first evidence for an association between neuronal pathology in dorsolateral prefrontal cortex and dopamine dysregulation in schizophrenia. These studies indicate that neuronal pathology of possible glutamatergic nature is correlated to lower subcortical dopamine activity at steady-state but, at the same time, to higher release of subcortical dopamine after 
a pharmacological stimulus is provided. One might also speculate that the prefrontally related dissociation between steady-state and stimulus-induced dopamine can clarify some aspects of negative and positive symptoms and of their coexistence in schizophrenia. In fact, previous data have suggested that lower dopamine activity in the prefrontal cortex can be associated with negative symptoms, whereas higher subcortical activity of dopamine can be associated with positive symptoms.

Since the data in patients are also limited by the fact that we have measured dopamine activity indirectly and we have also measured it in patients who had been previously treated with antipsychotics, we have tried to confirm the interpretation of our results in animal models of schizophrenia. Indeed, the results obtained in these further experiments are consistent with those obtained in patients with schizophrenia. These studies also add further complexity to the picture by showing that development of synaptic connections between prefrontal and hippocampal neurons may be critical for understanding dopamine dysregulation. In conclusion, our data in patients with schizophrenia and in an animal model of developmental prefrontal pathology indicate that activity in the striatal dopamine system is proportionate to the degree of dorsolateral prefrontal cortex neuronal deficit. This finding is consistent with current speculation that the dorsolateral prefrontal cortex has a critical downstream impact on the regulation of striatal dopaminergic function in this condition (Weinberger et al., 1986; 1995; Grace, 1991; Davis et al., 1991; Deutch, 1992).

\section{REFERENCES}

Abi-Dargham A.,Gil R., Krystal J., Baldwin R.M., Seibyl J.P., Bowers M., Van Dyck C.H., Charney D.S., Innis R.B. \& Laruelle, M (1998). Increased striatal dopamine transmission in schizophrenia: confirmation in a second cohort. American Journal of Psychiatry 155, 761-767.

Andreasen N.C., Flashman L., Flaum M., Arndt S., Swayze V., O'Leary D.S., Ehrhardt J.C. \& Yuh, W.T. (1994): Regional brain abnormalities in schizophrenia measured with magnetic resonance imaging. Journal of the American Medical Association 272(22), 1763-1769.

Bates T.E., Stranward M., Keelan J., Davey G.P., Munro P.M.G., \& Clark J.B (1996). Inhibition of $\mathrm{N}$-acetylaspartate production: implications for 1H MRS studies. Neuroreport 7, 1397 1400.

Bertolino A. Nawroz S., Mattay V.S., Duyn J.H., Moonen C.T.W., Barnett A.S., Frank J.A., Tedeschi G. \& Weinberger D.R (1996). A specific pattern of neurochemical pathology in schizophrenia as assessed by multislice proton magnetic reso- nance spectroscopic imaging. American Journal of Psychiatry $153,1554-1563$.

Bertolino A., Saunders R. C., Mattay V. S., Bachevalier J., Frank J. A. \& Weinberger D. R. (1997). Proton magnetic resonance spectroscopic imaging in monkeys with mesial temporo-limbic lesions. Cerebral Cortex 7, 740-748.

Bertolino A., Callicott J.H., Elman I., Mattay V.S., Tedschi G., Frank J.A., Breier A., \& Weinberger D.R (1998a). Regionally specific neuronal pathology in untreated patients with schizophrenia: a proton magnetic resonance spectroscopic imaging study. Biological Psychiatry 43, 641-648.

Bertolino A., Callicott J.H., Nawroz S., Mattay V.S., Duyn J.H., Tedwschi G., Frank J.A. \& Weinberger D.R (1998b). Reproducibility of proton magnetic resonance spectroscopic imaging in patients with schizophrenia. Neuropsychopharmacology $18,1-9$.

Bertolino A., Kumra S., Callicott J.H., Mattay V.S., Lestz R.M., Jacobsen L., Barnett I.S., Duyn J.H., Frank J.A., Rapoport J.L. \& Weinberger D.R. (1998c). Common pattern of cortical pathology in childhood-onset and adult-onset schizophrenia as identified by proton magnetic resonance spectroscopic imaging. American Journal of Psychiatry 155, 1376-1383.

Bertolino, A., Knable M.B., Saunders R.C., Callicott J.H., Kolachana B.S., Mattay V.S., Bachevalier J., Egan M.F., Frank J.A. \& Weinberger D.R (1999). The relationship between dorsolateral prefrontal $\mathrm{N}$-acetylaspartate measures and striatal dopamine activity in schizophrenia. Biological Psychiatry 45, 660-667.

Bertolino A., Breier A., Callicott J.H., Adler C., Mattay V.S., Shapiro M., Frank J.A., Pickar D. \& Weinberger D.R. (submitted for publication). The relationship between dorsolateral prefrontal neuronal $\mathrm{N}$-acetylaspartate and evoked release of striatal dopamine in schizophrenia.

Braff D.L., Heaton R., Kuck J., Cullum M., Moranville J., Grant I. \& Zisook S. (1991). The generalized pattern of neuropsychological deficits in outpatients with chronic schizophrenia with heterogeneous Wisconsin Card Sorting Test results. Archives of General Psychiatry 48(10), 891-898.

Braun A.R., Jaskiw G.E., Vladar K., Sexton R.H., Kolachana B.S. \& Weinberger D.R (1993). Effects of ibotenic acid lesion of the medial prefrontal cortex on dopamine agonist-related behaviors in the rat. Pharmacology Biochemistry and Behavior 46, 51-60.

Breier A., Su T.P., Saunders R., Carson R.E., Kolachana B.S., De Bartolomeis A., Weinberger D.R., Weisenfeld N., Malhotra A.K., Eckelman W.C. \& Pickar D. (1997). Schizophrenia is associated with elevated amphetamine-induced synaptic dopamine concentrations: evidence from a novel positron emission tomography method. Proceedings of the National Academy of Sciences of the USA 94, 2569-2574.

Buckley P.F., Moore C., Long H., Larkin C., Thompson P., Mulvany F., Redmond O., Stack J.P, Ennis J.T. \& Waddington J.L (1994). 1H Magnetic resonance spectroscopy of the left temporal and frontal lobes in schizophrenia: clinical neurodevelopmental and cognitive correlates. Biological Psychiatry 36, $792-800$.

Callicott J.H., Eegan M.F., Bertolino A., Mattay V.S., Langheim F.J.P, Frank J.A. \& Weinberger D.R (1998). Hippocampal Nacetylaspartate in unaffected siblings of patients with schizophrenia: a possible intermediate phenotype. Biological Psychiatry 44, 941-950.

Carlsson A (1998). The current status of the dopamine hypothesis of schizophrenia. Neuropsychopharmacology 1, 179-186. 
Clark J.B (1998). N-acetyl aspartate: a marker for neuronal loss or mitochondrial dysfunction. Developmental Neuroscience 20, 271-276

Davis K.L., Kahn R.S., Ko G. \& Davidson M (1991). Dopamine in schizophrenia: a review and reconceptualization American Journal of Psychiatry 148, 1474-1486.

Deicken R.F., Zhou L., Schuff N., Fein G. \& Weiner M.W (1998). Hippocampal neuronal dysfunction in schizophrenia as measured by proton magnetic resonance spectroscopy. Biological Psychiatry 43, 483-488.

Deutch A.Y (1992). The regulation of subcortical dopamine systems by the prefrontal cortex: interactions of central dopamine systems and the pathogenesis of schizophrenia. Journal of Neural Transmission Supplementum 36, 61-89.

Divac I., Kosmal A., Bjorkiund A. \& Lindvall O (1978). Subcortical projections to the prefrontal cortex in the rat as revealed by the horseradish peroxidase technique. Neuroscience 3,785 796.

Flores G., Wood G.K., Liang J.J., Quirion R. \& Srivastava L.K (1996). Enhanced amphetamine sensitivity and increased expression of dopamine $D 2$ receptors in postpubertal rats after neonatal excitotoxic lesions of the medial prefrontal cortex. Journal of Neuroscience 16, 7366-7375.

Frith C.D., Friston K.J., Herold S., Silbersweig D., Fletcher P., Cahill C., Dolan R.J., Frackowiak R.S.J. \& Liddle P.F. (1995). Regional brain activityin chronic schizophrenic patients during performance of a verbal fluency task. British Journal of Psychiatry 167, 343-349.

Goldberg T.E. \& Gold J.M. (1995). Neurocognitive deficits in schizophrenia. In Schizophrenia (ed. S.R. Hirsch and D.R. Weinberger), pp. 146-162. Blackwell Science: Oxford.

Grace A.A (1991). Phasic versus tonic dopamine release and the modulation of dopamine system responsivity: a hypothesis for the etiology of schizophrenia. Neuroscience 41, 1-24.

Hietala J., Syvalahti E. \& Vuorio K (1994). Striatal dopamine D2 receptor binding characteristics in neuroleptic naive schizophrenics studied with positron emission tomography. Archives of General Psychiatry 51, 116-123.

Jaskiw G.E., Karoum F.K. \& Weinberger D.R (1990). Persistent elevations in dopamine and its metabolites in the nucleus accumbens after mild subchronic stress in rats with ibotenic acid lesions of the medial prefrontal cortex. Brain Research 534, 321-323.

Jaskiw G.E. \& Weinberger D.R. (1992). Dopamine and schizophrenia: a cortically corrective perspective. Seminars in Neurosciences $41,179-188$

Karreman M. \& Moghaddam B. (1996). The prefrontal cortex regulates the basal release of dopamine in the limbic striatum: an effect mediated by ventral tegmental area. Journal of Neurochemistry 66(2), 589-598.

Knable M.B., Egan M.F., Heinz A., Gorey J., Lee K.S., Coppola R. \& Weinberger D.R (1997). Evidence for a relationship between altered dopaminergic function and negative symptoms in drug-free schizophrenic patients: an I-123 IBZM-SPECT study. British Journal of Psychiatry 171, 574-577.

Kornhuber J., Riederer P., Reynolds G.P., Beckmann H., Jellinger K. \& Gabriel E. (1989). 3H-spiperone binding sites in postmortem brains from schizophrenic patients: relationship to neuroleptic drug treatment, abnormal movements, and positive symptoms. Journal of Neural Transmission 75, 1-10.

Laruelle M., Abi-Dargham A., van-Dyck C.H., Gil R., D'Souza C.D., Erdos J., McKCance E., Rosenblatt W., Fingado C., Zoghbi S.S., Baldwin R.M., Seibyl J.P., Krystal J.H., Charney
D.S., \& Innis R.B (1996). Single-photon emission computerized tomography imaging of amphetamine-induced dopamine release in drug-free schizophrenic subjects. Proceedings of the National Academy of Science 93, 9235-9240.

Lee T., Seeman P., Toutellotte W.W., Farley I.J. \& Hornykiewicz O. (1978). Binding of 3H-neuroleptics and3H-apomorphine in schizophrenic brains. Nature 274, 897-900.

Lewis D.A. (1997). Development of prefrontal cortex during adolescence: insights into vulnerable neural circuits in schizophrenia. Neuropsychopharmacology 16, 385-398.

Maier M., Ron M.A., Barker G.J. \& Tofts P.S (1995). Proton magnetic resonance spectroscopy: an in vivo method of estimating hippocampal neuronal depletion in schizophrenia. Psychological Medicine 25, 1201-1209.

Moffett, J.R. \& Namboodiri, M.A (1995). Differential distribution of N-acetylaspartylglutamate and $\mathrm{N}$-acetylaspartate immunoreactivities in rat forebrain. Journal of Neurocytology 24, 409-433.

Moghaddam B. \& Adams B.W (1998). Reversal of phencyclidine effects by a group II metabotropic glutamate receptor agonist in rats. Science 281, 1349-1352.

Murase S., Grenhoff J., Chouvet G., Gonon F.G. \& Svensson T.H (1993). Prefrontal cortex regulates burst firing and transmitter release in rat mesolimbic dopamine neurons studied in vivo. Neuroscience Letters 157, 53-56.

Nasrallah H.A., Skinner T.E., Schmalbrock P. \& Robitaille P.M (1994). Proton magnetic resonance spectroscopy of the hippocampal formation in schizophrenia: a pilot study. British Journal of Psychiatry 165, 481-485.

Perrone-Bizzozzero N.I., Sower A.C., Bird E.D., Benowitz L.I., Ivins K.J. \& Neve R.L. (1996). Levels of the growth-associated protein GAP-43 are selectively increased in associattion cortices in schizophrenia. Proceedings of the National Academy of Sciences of the USA 93(24), 14182-14187.

Rajkowska G., Selemon L.D. \& Goldman-Rakic P.S. (1998). Neuronal and glial somal size in the prefrontal cortex. Archives of General Psychiatry 55, 215-224.

Renshaw P.F., Yurgelun-Todd D.A., Tohen M., Gruber S. \& Cohen B.M. (1995). Temporal lobe proton magnetic resonance spectroscopy of patients with first-episode psychosis. American Journal of Psychiatry 152, 444-446.

Roberts A.C., De Salvia M.A., Wilkinson L.S., Collins P., Muir J.L, Everitt B.J. \& Robbins T.W. (1994). 6-Hydroxydopamine lesions of the prefrontal cortex in monkeys enhance performance on an analog of the Wisconsin Card Sort Test: possible interactions with subcortical dopamine. Journal of Neuroscience 14, 2531-2544.

Rubin Y., LaPlaca M.C., Smith D.H., Thibault L.E. \& Lenkinski R.E (1995). The effect of $\mathrm{N}$-acetyl-aspartate on the intracellular calcium concentration in NTera2-neurons. Neuroscience Letters 198, 209-212.

Saunders R.C., Kolachana B., Bachevalier J. \& Weinberger D.R (1998). Neonatal lesions of the medial temporal lobe disrupt prefrontal cortical regulation of striatal dopamine. Nature $393,169-171$.

Schultz W., Dayan P. \& Montague P.R. (1997). A neural substrate for prediction and reward. Science 275, 1593-1599.

Selemon L.D., Rajkowska G. \& Goldman-Rakic P.S (1995). Abnormally high neuronal density in the schizophrenic cortex. A morphometric analysis of prefrontal area 9 and occipital area 17. Archives of General Psychiatry 52, 805-818.

Shim S.S., Bunney B.S. \& Shi W.X. (1996). Effects of lesions in the 


\section{A. Bertolino}

medial prefrontal cortex on the activity of midbrain dopamine neurons. Neuropsychopharmacology 15, 437-441.

Svensson T.H. \& Tung, C.S. (1989). Local cooling of prefrontal cortex induces pacemaker-like firing of dopamine neurons in rat ventral tegmental area in vivo. Acta Physiologica Scandinavica 136, 135-136.

Urenjak J., Williams S.R., Gadian D.G. \& Noble M. (1993). Proton nuclear magnetic resonance spectroscopy unambiguously identifies different neural cell types. Journal of Neuroscience 13, 981-989.
Weinberger D.R. (1987). Implications of normal brain development for the pathogenesis of schizophrenia. Archives of General Psychiatry 44, 660-669.

Weinberger D.R. \& Lipska, B.K. (1995). Cortical maldevelopment, antipsychotic drugs, and schizophrenia: a search for common ground. Schizophrenia Research 16, 87-110.

Weinberger D.R., Berman K.F. \& Zec R.F (1986). Physiological dysfunction of dorsolateral prefrontal cortex in schizophrenia: I. Regional cerebral blood flow (rCBF) evidence. Archives of General Psychiatry 43, 114-125. 